\title{
Controle postural e risco de quedas em pacientes com AVC agudo trombolisado
}

\author{
Postural control and fall risk in patients with acute \\ thrombolised stroke
}

\author{
Control postural y riesgo de caída en pacientes con acv \\ trombolizado agudo
}

\author{
Sandra Corradini ${ }^{1}$, Carla Ferreira do Nascimento ${ }^{2}$, Isabella Pereira \\ Rosa de Castro ${ }^{3}$, Daniele França dos Santos ${ }^{4}$, \\ Jorge Luis Motta dos Anjos ${ }^{5}$
}

\begin{abstract}
1.Fisioterapeuta, Doutoranda em Dança na Universidade Federal da Bahia, Residente no Programa de Residência Multiprofissional em Neurologia, Salvador-BA, Brasil. https://orcid.org/0000-0003-2101-0091 2.Fisioterapeuta, Doutora em Saúde Pública pela Universidade de São Paulo, Serviço de Preceptora no Programa de Residência de Fisioterapia Neurofuncional e Fisioterapeuta no Hospital Roberto Santos, Salvador-BA, Brasil. https://orcid.org/0000-0002-0054-277X

3.Fisioterapeuta, Mestre em Ciências da Saúde pela Universidade Federal da Bahia, Preceptora e tutora no Programa de Residência de Fisioterapia Neurofuncional, Hospital Geral Roberto Santos, Salvador-BA, Brasil. ORCID: 0000-0001-9519-6162. https://orcid.org/0000-0001-9519-6162

4.Fisioterapeuta, Especialista em Terapia Intensiva Adulto e em Fisioterapia Neurofuncional pelo COFFITO, Preceptora e tutora no Programa de Residência de Fisioterapia Neurofuncional, Hospital Geral Roberto Santos, Salvador-BA, Brasil. https://orcid.org/0000-0001-8440-3883

5.Fisioterapeuta, doutorando em Medicina e Saúde Humana pela Universidade Federal da Bahia, Coordenador de Ensino do Hospital Geral Roberto Santos, Salvador-BA, Brasil. https://orcid.org/00000003-2897-9858
\end{abstract}

\section{Resumo}

Introdução. Pacientes com acidente vascular cerebral isquêmico (AVCI) agudo trombolisado, internados em unidade de AVC, tendem a apresentar déficits de controle postural (CP) que impactam na mobilidade funcional (MF) e aumentam o risco de quedas (RQ). Objetivos. Verificar a associação entre CP e MF e identificar os principais domínios e tarefas do CP que impactam na mobilidade independente. Método. Estudo transversal, realizado com 34 pacientes consecutivos, com deambulação independente. CP e MF foram mensurados pelos instrumentos PASS e TUG, respectivamente, e analisados pelo teste de correlação de Pearson. A PASS, os domínios manutenção de postura (MAP) e mudança de postura (MUP), e as tarefas da PASS foram comparadas ao TUG utilizando, respectivamente, os testes ANOVA e quiquadrado, e o RQ foi avaliado pelo TUG. Os dados foram analisados utilizando o programa SPSS 17.0, $a<0,05$ e poder de $80 \%$. Resultados. Correlação de Pearson negativa moderada para PASS e TUG $(r=-0,576 ; p=0,000)$, fraca para MAP e TUG $(r=-0,448 ; p=0,049)$ e moderada para MUP e TUG $(r=-0,641 ; p=0,006)$. As tarefas que mais impactaram na MF foram em pé sobre a perna não hemiparética $(r=-0,674 ; p=0,000)$ e em pé sobre a perna não hemiparética $(r=-0,631 ; p=0,000)$. As tarefas sentado sem apoio $(p=0,055)$ e de decúbito dorsal para o lado afetado $(p=0,058)$ não tiveram significância estatística. Os pacientes apresentaram de moderado a alto RQ. Conclusão. CP impacta na MF em pacientes com mobilidade preservada e comprometida, apresentando maior implicação do CP estático e maior comprometimento em condição unipodal e maior RQ nestes pacientes.

Unitermos. Equilíbrio postural; Limitação da mobilidade; Acidente vascular cerebral; Fase aguda; Terapia trombolítica; Assistência hospitalar

\footnotetext{
Abstract

Introduction. Patients with thrombilized acute stroke, admitted to a stroke unit, tend to have deficits in postural control (PC) that impact on functional mobility (FM) and increase the risk of falls (RF). Obkective. Check the association between PC and FM and identify the main domains and tasks of the PC that impact on independent mobility. Method. Cross-sectional
} 
study, carried out with 34 consecutive patients, with independent walking. PC and FM were measured by the PASS and TUG instruments, respectively, and prevented by the Pearson correlation test. PASS, the posture maintenance (MAP) and posture change (MUP) domains, and PASS tasks were compared to the TUG using the ANOVA and chi-square tests, respectively, and the RF was assessed by the TUG. The data were analyzed using the SPSS 17.0 program, $\mathrm{a}<0.05$ and $80 \%$ power. Results. Moderate negative Pearson correlation for PASS and TUG $(r=-0.576 ; p=0.000)$, weak for MAP and TUG $(r=-0.448 ; p=0.049)$ and moderate for MUP and TUG $(r=-0.641 ; p=0.006)$. The tasks that most impacted FM were standing on the nonhemiparetic leg $(r=-0.674 ; p=0.000)$ and standing on the non-hemiparetic leg $(r=-0.631$; $p=0.000)$. The tasks seated without support $(p=0.055)$ and supine for the affected side $(p=0.058)$ were not statistically significant. Patients presented moderate to high RF. Conclusion. PC impacts on FM in patients with preserved and compromised mobility, with greater implication of static PC and greater impairment in unipodal condition and greater RF in these patients.

Keywords. Postural balance; Limitation of mobility; Stroke; Acute phase; Thrombolytic therapy; Hospital assistance

\section{Resumen}

Introducción. Los pacientes con accidente cerebrovascular isquêmico agudo trombilizado, ingresados en una unidad de unidad de accidente cerebrovascular, suelen presentar déficits en el control postural (PC) que repercuten en la movilidad funcional (MF) y aumentan el riesgo de caídas (RC). Objetivo. Verificar la asociación entre PC y MF, y identificar los principales dominios y tareas del PC que influyen en la movilidad independiente. Método. Estudio transversal, realizado con 34 pacientes consecutivos, con marcha independiente. CP y MF fueron medidos por los instrumentos PASS y TUG, respectivamente, y prevenidos por la prueba de correlación de Pearson. PASS, los dominios de mantenimiento de la postura (MAP) y cambio de postura (MUP), y las tareas de PASS se compararon con el TUG utilizando las pruebas ANOVA y chi-cuadrado, respectivamente, y el RQ se evaluó mediante el TUG. Los datos fueron analizados utilizando el programa SPSS 17.0 , $a<0.05$ y potencia del $80 \%$. Resultados. Correlación de Pearson negativa moderada para PASS y TUG $(r=-0,576 ; p=0,000)$, débil para MAP y TUG $(r=-0,448 ; p=0,049)$ y moderada para MUP $(r=-0,641 ; p=0,006)$. Las tareas que más afectaron a la FM fueron estar de pie sobre la pierna no hemiparética $(r=-0,674 ; p=0,000)$ y pararse sobre la pierna no hemiparética $(r=-0,631 ; p=0,000)$. Las tareas sentado sin apoyo $(p=0,055)$ y supino para el lado afectado $(p=0,058)$ no fueron estadísticamente significativas. Pacientes presentaron moderado a alto RC. Conclusión. La CP impacta en la MF en pacientes con movilidad preservada y comprometida, con mayor implicación de PC estática y mayor deterioro en condición unipodal y mayor RC en estos pacientes.

Palabras clave. Equilibrio postural; Limitación de movilidad; Accidente cerebrovascular; Fase aguda; Terapia trombolítica; Asistencia hospitalaria

Trabalho realizado na Universidade Federal da Bahia, Salvador-BA, Brasil.

Endereço para correspondência: Sandra Corradini. Av. Cardeal da Silva 2210, Edifício Villa da Sereia, apto 204B. Rio Vermelho. Salvador-BA, Brasil. Cep 41950-495. E-mail: fisio.scorr@gmail.com

\section{INTRODUÇÃO}

\section{Controle postural (CP) é uma habilidade complexa} baseada na interação de múltiplos processos sensoriomotores e demanda ação coordenada dos sistemas motor, visual, vestibular e somatosensorial, envolvendo ajustes posturais antecipatórios (feedforward) e reativos 
(feedback sensorial) diante de perturbações externas previsíveis e inesperadas do ambiente, respectivamente ${ }^{1}$. Por definição, CP é a habilidade de assumir ou manter a postura desejada durante uma atividade estática ou dinâmica² e seu objetivo é o equilíbrio e a orientação postural ${ }^{1}$. Do ponto de vista biomecânico, o corpo deve estar posicionado com o centro de gravidade (CG) dentro dos limites da base de sustentação e adequar a estabilidade de tronco para livre movimentação dos membros apendiculares ${ }^{3}$.

O CP em pacientes com acidente vascular cerebral (AVC) é frequentemente associado a déficits de mobilidade ${ }^{3}$, cujos componentes são descritos como mudanças de posição, manobras de equilíbrio e marcha ${ }^{4}$. Como habilidade para se movimentar de um lugar para o outro sem necessitar de auxílio ${ }^{5}$, a mobilidade funcional (MF) requer habilidades básicas, como se deitar e levantar da cama, sentar e levantar da cadeira, entrar e sair de um banheiro, e andar alguns metros $^{6}$. São manobras funcionais básicas que permitem a realização das atividades de vida diárias (AVDs) com eficiência e segurança. Prejuízos na MF aumentam a dependência nas AVDs e o RQ ${ }^{3,7}$.

Indivíduos com AVC apresentam risco aumentado de quedas em todas as fases pós-AVC ${ }^{8}$. Aspectos multifatoriais estão relacionados à etiologia das quedas tanto no AVC agudo como crônico, dentre eles, a perda de equilíbrio por alterações no $\mathrm{CP}^{3}$. Comparado a outras patologias, o RQ em pacientes com AVC agudo (1-7 dias pós-ictus) em ambiente 
hospitalar foi identificado como sendo mais que o dobro do que em pacientes internados com insuficiência cardíaca congestiva (ICC) e pneumonia adquirida na comunidade (PAC), consistindo em uma das maiores ameaças à segurança do paciente com $\mathrm{AVC}^{8}$.

O AVC isquêmico (AVCI) é responsável por $85 \%$ dos casos de $A_{V C}$. Pacientes com AVCI agudo podem se beneficiar da trombólise quando atendidos os critérios de elegibilidade e trombolisados em até quatro horas e meia após o ictus ${ }^{10}$. Trombólise é a dissolução de um trombo intravascular com ativador do plasminogênio tissular (rTPA), medicamento endovenoso, utilizado para restaurar o fluxo sanguíneo cerebral. O rTPA não reduz a mortalidade de forma direta, mas é efetivo para melhora da gravidade neurológica e redução das incapacidades funcionais ${ }^{11}$.

A terapia trombolítica vem sendo utilizada em quantidade crescente no Brasil desde a sua liberação pelo Ministério da Saúde em 2001'12. Em Salvador, especificamente, no período de 2009 a 2019, dados do Sistema de Informações Hospitalares do Sistema Único de Saúde (SIH/SUS), referentes ao hospital em que foi realizado o presente estudo, apontam que $8,24 \%$ dos casos de internação por AVC foram autorizados para uso de trombolítico, atingindo uma média de 54,25 trombólises anuais em 10 anos.

Considerando a tendência no aumento de pacientes trombolisados diante da atual transição epidemiológica, com aumento de doenças crônicas não transmissíveis no Brasil e 
no mundo ${ }^{13}$, e a importância de um adequado CP para um desfecho satisfatório da MF que garanta a máxima independência possível na alta hospitalar, este estudo teve como objetivo verificar a associação entre CP e MF em pacientes com AVCI agudo trombolisados, internados em UAVC, e identificar os principais domínios e tarefas do CP que impactam na mobilidade independente e contribuem para 0 aumento do RQ nessa população.

\section{MÉTODO}

\section{Amostra}

Trata-se de estudo observacional, do tipo transversal, realizado com pacientes com AVCI agudo trombolisados, internados em UAVC de um hospital público de grande porte, referência em neurologia no estado da Bahia, Brasil.

A amostra foi composta de 34 pacientes com AVCI agudo trombolisados, avaliados em até sete dias após exame admissional em UAVC, internados no período de março de 2019 a dezembro de 2020.

Este estudo fez parte de um projeto de pesquisa mais amplo em curso, aprovado pelo Comitê de Ética e Pesquisa do referido hospital, e está de acordo com as recomendações contidas na Resolução CNS 466/2012 (CONEP), do Ministério da Saúde, sob o número de registro CAAE 87271218.0.0000.5028. 


\section{Procedimento}

O recrutamento foi feito por meio da amostragem acessível e consecutiva. Todos foram convidados a participar como voluntários e informados sobre os objetivos e procedimentos do estudo, e assinaram o Termo de Consentimento Livre e Esclarecido (TCLE).

Os critérios de inclusão foram: (1) diagnóstico de AVCi com trombólise sem evidência hemorrágica confirmada em tomografia computadorizada de crânio de controle e pela equipe médica; (2) idade igual ou superior a 18 anos; (3) nível de consciência satisfatório para responder a comandos, aferido por meio da escala National Institutes of Health Stroke Scale (NIHSS, itens $1 \mathrm{a}=0-1$ e $1 \mathrm{c}=0$ ); (4) ausência de incapacidade significante prévia, referido na aplicação da Escala de Rankin modificada $(\mathrm{mRS}<2) ; \quad$ (5) limites fisiológicos satisfatórios (PAS 110-220 mmHg; SpO2>92\% sem uso de 02 suplementar, $\mathrm{FC} 40-110 \mathrm{mmHg}, \mathrm{T}<38^{\circ}, 5 \mathrm{C}$ ). Os critérios de exclusão foram: (1) outra doença grave (2) coronariopatia, (3) afasia; (4) instabilidade hemodinâmica; (5) hipotensão postural durante os procedimentos (queda de PAS $\geq 20 \mathrm{mmHg}$ e/ou $P A D \geq 10 \mathrm{mmHg}$ três minutos após ortostase em relação aos valores com o paciente sentado).

Variáveis e mensuração dos desfechos

Dados demográficos de sexo e idade, dados clínicos e resultados obtidos mediante a aplicação das escalas NIHSS e mRS foram registrados em ficha clínica e utilizados para caracterização da amostra. Os dados de CP e de MF foram 
mensurados por meio da Postural Assessment Scale for Stroke Patients (PASS) e do teste Timed Up and Go (TUG), respectivamente.

A NIHSS é um instrumento de avaliação quantitativa, utilizado internacionalmente para determinar o nível de comprometimento neurológico em vítimas de AVC, sendo um indicador da gravidade da lesão. A escala compreende 11 itens, mensuráveis de acordo com o déficit apresentado, e sua pontuação varia de 0 a 42 pontos, sendo que uma pontuação maior indica maior gravidade de lesão ${ }^{14}$. A NIHSS é uma escala validada para a população brasileira ${ }^{15}$ e a categorização da amostra para esta variável foi definida segundo estudo AVERT ${ }^{16}$, adotando os escores NIHSS 1-7 como déficit leve, NIHSS 8-16 como déficit moderado e NIHSS 17-21 como déficit grave. Pacientes com NIHSS 0 foram considerados sem déficit neurológico. Pacientes com grande risco de hemorragia intracraniana associada ao tratamento trombolítico corresponderam a NIHSS $>22$.

A $m R S$ tem como objetivo mensurar o grau de incapacidade e dependência nas AVDs em vítimas de AVC. Em sua apresentação original, foi dividida em seis graus, onde o grau zero corresponde a indivíduos sem sintomas residuais ou incapacidade e o grau cinco a indivíduos com incapacidade grave, restritos ao leito ou à cadeira ${ }^{17}$. Posteriormente, foi acrescentado o grau seis, correspondendo à morte ${ }^{18}$. No presente estudo, os valores obtidos nesta escala foram caracterizados segundo estudo AVERT ${ }^{16}$, adotando os escores mRS 0-3 e mRS 4-6, 
indicando, respectivamente, prognóstico favorável e ruim aos 3 meses após AVC.

A PASS foi o instrumento utilizado para avaliação do CP. A PASS é uma escala de desempenho postural, desenvolvida para pacientes com $\mathrm{AVC}^{19}$ e validada para a população brasileira $^{20}$. A PASS avalia e monitora O CP em pacientes após o AVC por meio de 12 tarefas com dificuldade crescente, dispostos em dois domínios, nas posturas deitada, sentada e em pé. O primeiro domínio é constituído pelos primeiros cinco itens e avalia a manutenção da postura (MAP), enquanto o segundo domínio é composto por sete itens e avalia a mudança de postura (MUP). A pontuação total varia de zero a 36 e cada tarefa varia de zero a três pontos, sendo que zero significa incapacidade de realizar a tarefa e três indica a capacidade de realizá-la sem auxílio. As pontuações máximas do primeiro e do segundo domínios são, respectivamente, 15 e 21 . Quanto mais alta a pontuação total, melhor o CP. O CP, os diferentes domínios, bem como cada tarefa da PASS, foram mensurados e considerados variáveis quantitativas.

O instrumento utilizado para avaliar o RQ foi o TUG. Este instrumento avalia a MF de forma quantitativa por meio da mensuração do tempo utilizado pelo paciente para se levantar de uma cadeira padrão, a partir da posição sentada/encostada, caminhar três metros à frente, virar-se, caminhar de volta e sentar-se novamente na cadeira, em ritmo auto selecionado e seguro. Este teste reúne um conjunto de ações tipicamente rotineiras, fundamentais para 
a mobilidade independente, de modo que o desempenho no teste é afetado pelo tempo de reação, força muscular dos membros inferiores, equilíbrio e velocidade da marcha ${ }^{20}$. Nenhum dispositivo de auxílio de marcha foi utilizado.

No presente estudo, os resultados obtidos no TUG foram considerados preditores para quedas, sendo um teste indicado para idosos saudáveis e com outras patologias, incluindo o $\mathrm{AVC}^{3}$. O ponto de corte adotado foi de $14 \mathrm{~s}$, sendo considerado baixo RQ valores iguais e inferiores a $14 \mathrm{~s}$, indicando mobilidade preservada, e valores superiores a $14 \mathrm{~s}$, considerados de moderado a alto $R Q$, indicando mobilidade comprometida. Os valores do TUG foram categorizados a partir de estudo realizado com pacientes internados em UAVC ${ }^{21}$.

\section{Análise estatística}

Os dados foram analisados estatisticamente no programa SPSS 17.0, utilizando $\mathrm{p}<0,05$ e poder estatístico de $80 \%$. As variáveis categóricas descreveram o perfil da amostra e foram analisadas por meio de estatística descritiva.

A distribuição normal da amostra foi verificada por meio do teste Kolmogorov-Smirnov. O teste de correlação linear de Pearson foi utilizado para analisar o coeficiente de correlação ( $r$ ) entre as variáveis CP e MF. A correlação foi classificada como insignificante $(0,00$ a $-0,30)$, fraca $(-0,30$ a $-0,50)$, moderada $(-0,50$ a 0,70$)$, forte $(-0,70$ a $-0,90)$ e muito forte $(-0,90 \text { a }-1,00)^{22}$. 
As médias dos domínios MAP e MUP da PASS foram comparadas por meio do teste ANOVA, sendo utilizado o TUG categorizado de acordo com o RQ de 14s. O teste quiquadrado foi utilizado para verificar a associação de cada tarefa da PASS ao TUG ordinal, e o coeficiente de Pearson verificou o nível de intensidade da associação entre as variáveis, bem como a associação entre CP e NIHSS, e CP e Rankin. O RQ foi avaliado a partir da variável TUG, categorizada em baixo $\mathrm{RQ}(\leq 14 \mathrm{~s})$ e moderado-alto $\mathrm{RQ}$ $(>14 s)$.

\section{RESULTADOS}

A amostra foi composta de 34 pacientes, sendo $50 \%$ do sexo masculino e $50 \%$ do sexo feminino, com média de idade aproximada de 58 anos, variando entre idade mínima de 25 anos e máxima de 87 anos. Quase a totalidade dos pacientes $(97,1 \%)$ teve AVC pela primeira vez e todos relataram independência prévia. No período de até sete dias, 50\% dos pacientes tiveram alta hospitalar. No momento da avaliação, $44,1 \%$ dos pacientes apresentaram AVC leve e 55,9\% não apresentaram déficit neurológico, e 94,1\% não apresentaram incapacidade ou apresentaram incapacidade significante, leve ou moderada. As características gerais da amostra são apresentadas na Tabela 1.

Os escores obtidos na PASS e no TUG foram submetidos à análise por meio do teste de correlação linear de Pearson, que mostrou correlação linear negativa moderada para estas variáveis $(r=-0,576 ; p=0,000)$ (Gráfico 1$)$. 
Tabela 1. Caracterização da amostra $(n=34)$.

\begin{tabular}{|c|c|c|}
\hline \multirow{3}{*}{$\begin{array}{l}\text { Variáveis } \\
\text { Sexo }\end{array}$} & & n (\%) \\
\hline & Feminino & $17(50,0)$ \\
\hline & Masculino & $17(50,0)$ \\
\hline Idade (anos) & & $58,03 \pm 13,805(25-87) *$ \\
\hline \multirow[t]{7}{*}{ Doenças cardiovasculares } & Hipertensão arterial & $26(76,5)$ \\
\hline & Diabetes mellitus & $8(23,5)$ \\
\hline & Dislipidemia & $3(8,8)$ \\
\hline & Fibrilação atrial & $3(8,8)$ \\
\hline & Doença arterial coronariana & $1(2,9)$ \\
\hline & Insuficiência congestiva cardíaca & $1(2,9)$ \\
\hline & Sem doenças cardiovasculares & $7(20,6)$ \\
\hline \multirow[t]{3}{*}{ Etilismo } & Etilista/abstêmico & $15(44,2)$ \\
\hline & Não etilista & $13(38,2)$ \\
\hline & Não informado & $6(17,6)$ \\
\hline \multirow[t]{2}{*}{ Tabagismo } & Fumante/ex-fumante & $12(35,3)$ \\
\hline & Nunca fumou & $22(64,7)$ \\
\hline AVC Prévio & Isquêmico & $1(2,9)$ \\
\hline Rankin prévio & Independente & $34(100,0)$ \\
\hline \multirow[t]{3}{*}{ Hemisfério da lesão } & Direito & $13(38,2)$ \\
\hline & Esquerdo & $17(50)$ \\
\hline & Não informado & $4(11,8)$ \\
\hline \multirow[t]{2}{*}{$\mathrm{AH}$} & & $6,06 \pm 1,217(4-7)^{*}$ \\
\hline & $\leq 7$ dias & $17(50)$ \\
\hline \multirow[t]{2}{*}{ NIHSS ( $\leq 7$ dias) } & Sem déficit (NIHSS 0) & $19(55,9)$ \\
\hline & Déficit leve (NIHSS 1-7) & $15(44,1)$ \\
\hline \multirow[t]{2}{*}{ Rankin ( $\leq 7$ dias) } & Sem incapacidade (mRS 0) & $18(52,9)$ \\
\hline & Incapacidade leve/moderada (mRS 1-3) & $16(47,1)$ \\
\hline
\end{tabular}

AVC - acidente vascular cerebral; AH - avaliação hospitalar; NIHSS - National Institutes of Health Stroke Scale; n(\%) - frequência absoluta (frequência percentual). * média DP (mín.-máx.). 
Gárico 1. Correlação de Pearson para as variáveis PASS e TUG $(n=34)$.

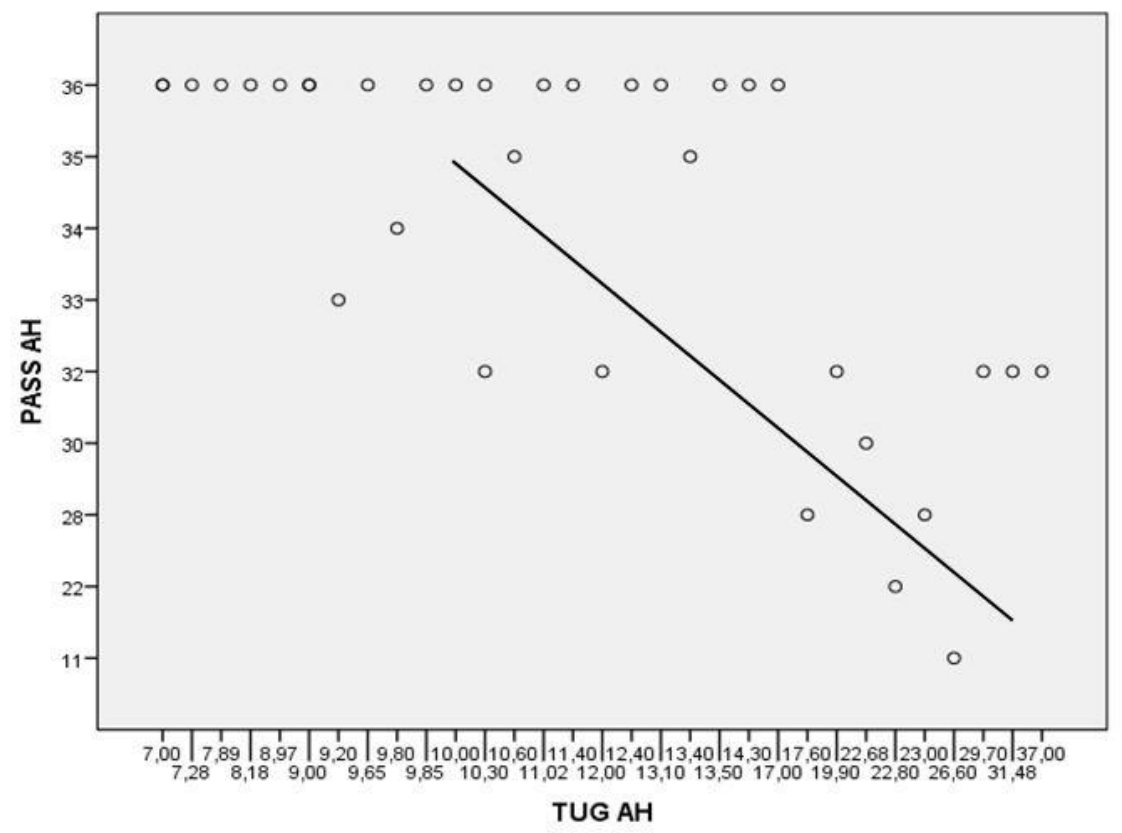

As médias das variáveis MAP e MUP foram comparadas, utilizando-se o TUG categorizado de acordo com o RQ. A

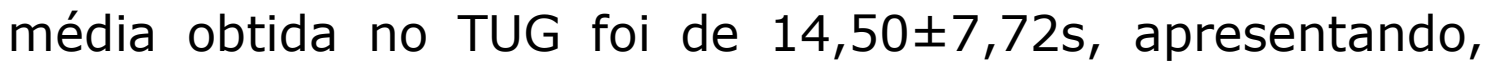
respectivamente, uma variação mínima e máxima de sete e 37 segundos. Os resultados são apresentados na Tabela 2.

Tabela 2. Análise comparativa das variáveis MAP e MUP $(n=34)$.

\begin{tabular}{lccccc}
\hline Variáveis & $\mathbf{n}(\%)$ & TUG & M $\pm \mathbf{D P}$ & p-valor & $\mathbf{r}$ \\
\hline MAP & $23(67,65)$ & $\leq 14$ & $14,35 \pm 1,301$ & $0,006 *$ & $-0,641$ \\
& $11(32,35)$ & $>14$ & $10,82 \pm 3,241$ & & \\
\multirow{2}{*}{ MUP } & $23(67,65)$ & $\leq 14$ & $20,87 \pm 0,626$ & $0,049 *$ & $-0,448$ \\
& $11(32,35)$ & $>14$ & $18,18 \pm 3,341$ & &
\end{tabular}

MAP - manutenção de postura; MUP - mudança de postura; n (\%) - frequência absoluta (frequência percentual); TUG - Time Up and Go; M DDP - média \pm desvio padrão; $p$-valor - nível de significância; $r$ - coeficiente de correlação de Pearson. * nível de significância estatística $<0,05$. 


\section{A Tabela 3 apresenta os valores resultantes das análises dos dados obtidos na PASS, nos domínios MAP e MUP, bem como nas tarefas da PASS.}

Tabela 3. Frequência, média e desvio padrão, e valores máximo e mínimo da PASS, MAP, MUP e tarefas da PASS, e associações entre cada tarefa da PASS e TUG, nível de significância e força de correlação de Pearson $(n=34)$.

\begin{tabular}{|c|c|c|c|c|c|}
\hline Variáveis & $\mathbf{n}$ & M $\pm D P$ & Min-Max & p-valor & $\mathbf{r}$ \\
\hline PASS & 34 & $33,29 \pm 5,066$ & $11-36$ & 0,000 & $-0,576^{2}$ \\
\hline MAP & 34 & $13,21 \pm 2,71$ & 3-15 & 0,006 & $-0,641^{2}$ \\
\hline MUP & 34 & $20,00 \pm 2,58$ & $8-21$ & 0,049 & $-0,448^{1}$ \\
\hline \multicolumn{6}{|l|}{ Tarefas do domínio MAP } \\
\hline Sentado sem apoio & 34 & $2,97 \pm 0,171$ & $2-3$ & 0,486 & $-0,124$ \\
\hline Em pé com apoio & 33 & $2,91 \pm 0,514$ & $0-3$ & 0,113 & $-0,277$ \\
\hline Em pé sem apoio & 33 & $2,85 \pm 0,558$ & $0-3$ & $0,029 *$ & $-0,3741$ \\
\hline Em pé sobre a perna não hemiparética & 32 & $2,32 \pm 1,007$ & $0-3$ & $0,000 *$ & $-0,631^{2}$ \\
\hline Em pé sobre a perna hemiparética & 31 & $2,15 \pm 1,007$ & $0-3$ & $0,000 *$ & $-0,674^{2}$ \\
\hline \multicolumn{6}{|l|}{ Tarefas do domínio MUP } \\
\hline De pé para sentado & 33 & $2,79 \pm 0,592$ & $0-3$ & $0,012 *$ & $-0,427^{1}$ \\
\hline De sentado para supina & 31 & $2,91 \pm 0,288$ & $2-3$ & $0,021 *$ & $-0,394^{1}$ \\
\hline De supina para o lado afetado & 32 & $2,94 \pm 0,239$ & $2-3$ & 0,113 & $-0,277$ \\
\hline De supina para o lado não afetado & 33 & $2,97 \pm 0,171$ & $2-3$ & 0,053 & $-0,335^{1}$ \\
\hline De decúbito para sentado & 32 & $2,94 \pm 0,239$ & $0-3$ & 0,053 & $-0,335^{1}$ \\
\hline De sentado para a posição de pé & 33 & $2,79 \pm 0,592$ & $0-3$ & $0,007^{*}$ & $-0,452^{1}$ \\
\hline Em pé, apanhar uma caneta do chão & 32 & $2,74 \pm 0,751$ & $0-3$ & $0,006 *$ & $-0,460^{1}$ \\
\hline
\end{tabular}

PASS - Postural Assessment Scale for Stroke Patients; MAP - manutenção de postura; MUP - mudança de postura; $\mathrm{n}$ frequência absoluta; $M \pm D P$ - média \pm desvio padrão; Min-Max - mínimo-máximo; $p$-valor = nível de significância; $r=$ coeficiente de correção de Pearson. *nível de significância estatística $<0,05 ;{ }^{1}$ correlação fraca; ${ }^{2}$ correlação moderada. 
do teste de correlação de Pearson, mostrando fraca correlação negativa entre PASS e NIHSS ( $r=-0,472$; $p=0,005)$ e forte correlação negativa entre PASS e Rankin $(r=-0,711 ; p=0,000)$.

\section{DISCUSSÃO}

\section{Controle postural e mobilidade funcional}

Este estudo verificou a associação entre CP e o MF por meio da PASS e do TUG de 34 pacientes com AVCI agudo trombolisados, avaliados em uma média de seis dias após o ictus, em uma UAVC. Todos os pacientes tinham marcha independente e apresentaram média na PASS de 33,29, com variação de 11 a 36, e tempo médio no TUG de 14,50s, o que indica que a amostra apresentou, em média, de moderado a alto RQ. O teste de correlação de Pearson verificou correlação negativa moderada entre as variáveis PASS e TUG, mostrando que quanto maior pontuação na PASS, menor é o tempo do TUG.

Os achados no presente estudo são concordantes aos encontrados na literatura acerca da implicação das alterações do $\mathrm{CP}$ na $\mathrm{MF}$ em pacientes com $\mathrm{AVC}^{3,21,23}$. Entretanto, foram encontrados poucos estudos que utilizaram a PASS como instrumento de avaliação no ambiente hospitalar e muitas evidências acerca do CP e MF em pacientes com AVC crônico.

O estudo de validação da versão brasileira da PASS ${ }^{20}$ verificou a média de 28,26 para a PASS total de 19 pacientes, avaliados em uma média de 55 meses pós-AVC, e nenhum 
paciente alcançou a pontuação máxima de 36 pontos. Por outro lado, Benaim et al. ${ }^{19}$ compararam 30 indivíduos saudáveis a 58 pacientes 30 e 90 dias pós-AVC, encontrando uma pontuação média da PASS de 35,7, para indivíduos saudáveis, com variação de 32 a 36 .

Nosso estudo mostrou um escore médio na PASS total situado em uma faixa entre as médias obtidas nas duas citadas pesquisas. Embora 15 pacientes em nosso estudo não tenham atingido o escore máximo, é importante ressaltar a comparação feita entre os nossos valores da PASS e os valores obtidos no estudo original, relativos aos indivíduos saudáveis, acima descritos, com intuito de destacar a rápida melhora do $\mathrm{CP}$ dos pacientes no presente estudo. Este aspecto observado talvez possa ser parcialmente justificado pela terapia trombolítica, por favorecer a melhora dos desfechos funcionais na alta hospitalar.

O gráfico de correlação de Pearson permitiu observar um efeito teto importante na PASS, dado o alto número de pacientes que obteve a pontuação total de 36 pontos. Na amostra estudada, 19 pacientes alcançaram o escore total e apenas um teve a menor pontuação de 11 pontos, o que indica que pacientes que andam tendem a ter pontuação máxima na PASS.

No que tange a este aspecto, nosso estudo diverge do estudo de validação brasileira da $\operatorname{PASS}^{20}$, que não encontrou efeito teto da escala. Ainda difere do estudo de validação da PASS, que apontou efeito teto moderado após 90 dias pós- 
AVC $^{19}$, bem como do estudo que verificou efeito chão aos 14 dias e efeito teto significante aos 90 e 180 dias pós-AVC ${ }^{24}$. Esses diferenciais entre nossa pesquisa e os citados estudos sugerem que pode haver uma limitação da capacidade deste instrumento na discriminação dos pacientes trombolisados na fase aguda do AVCI.

\section{Controle postural estático e dinâmico e mobilidade preservada e comprometida}

Quedas em pacientes após AVC recebem considerável atenção na literatura, mostrando-se associadas a transferências em pacientes internados na fase aguda e à perda de equilíbrio durante a durante a marcha em indivíduos após AVC, viventes em comunidade, consistindo no fator de risco mais relatado em relação às AVDs, relacionado à mobilidade?.

A comparação das médias dos domínios MAP e MUP permitiu observar maior significância estatística e associação moderada para o domínio MAP e fraca associação e significância estatística para o domínio MUP. O CP estático teve maior impacto na $M F$, de modo que $67,65 \%$ apresentaram um MAP médio de 14,35, realizando o TUG em tempo inferior ou igual a 14s, indicando mobilidade preservada. Os demais 32,35\% da amostra apresentou a média 10,82 no MAP e realizaram o TUG em tempo superior a 14s, indicando mobilidade comprometida.

Em contrapartida, as médias obtidas para a variável MUP, para esses dois grupos de pacientes, mostraram-se 
ligeiramente abaixo da pontuação máxima para esse domínio e tiveram menor impacto na mobilidade. Considerando que a máxima pontuação do domínio MAP é 15 , foi possível observar que o $\mathrm{CP}$ estático impactou mais na mobilidade dos pacientes que apresentaram mobilidade comprometida (TUG>14s).

Em estudo recente realizado com pacientes com AVC, incluindo PASS e TUG, foram observados influência do CP no equilíbrio e na marcha e nível de correlação negativa moderada entre ambos os domínios da PASS e o TUG ${ }^{23}$. Outro estudo não verificou correlação entre as mesmas variáveis, mas apontaram as médias 11,1 para o MAP e 17,15 para o MUP20. Ambos os estudos incluíram pacientes com AVCI e AVCH crônicos. Tais resultados diferem do presente estudo, no que tange à seleção amostral e à fraca correlação encontrada entre as variáveis MUP e TUG. Isso sugere que a PASS mostra-se sensível às diferenças na seleção amostral, em especial, às características tipo de AVC e tempo de lesão.

Poucos estudos avaliaram a mobilidade por meio do TUG em pacientes com AVC agudo internados em UAVC. O ponto de corte de $14 \mathrm{~s}$ para RQ foi adotado a partir do estudo de Andersson et al. ${ }^{21}$ que avaliou exclusivamente pacientes internados em UAVC. A avaliação foi feita em uma média de oito dias pós-AVC, com o objetivo de verificar a capacidade desse instrumento para identificar o $R Q$, identificando tempo igual ou inferior a esse valor para não caidores. 
Foi verificado excelente confiabilidade do TUG por meio da avaliação de 60 pacientes com AVC internados em UAVC, reportando quatro médias, entre a máxima de $16,60 \mathrm{~s}$ e mínima de 15,40s, obtidas em duas sessões de testes, realizadas em dois dias consecutivos, com avaliações feitas em uma mediana de 5 dias após AVC 25.

A diferença na média do TUG em nosso estudo das encontradas nas citadas pesquisas pode estar relacionada ao diferencial na seleção amostral. Outros estudos ou não especificaram o tipo de $A V C^{21}$, ou incluíram pacientes com AVCH $(9,7 \%)^{25}$ e reportaram o uso de dispositivo auxiliar de marcha por $69,2 \%$ da amostra.

Nossos resultados confirmam o tipo de AVC e mostram o uso de recursos auxiliares da marcha como fatores diferenciais importantes na avaliação da mobilidade em pacientes com AVC agudo. O AVCH apresenta déficits neurológicos mais graves que o infarto cerebral em estágio inicial agudo e ganhos funcionais mais expressivos do AVCI foram reportados 30 dias após reabilitação, iniciada mediante triagem feita 5-7 dias após o $\mathrm{AVC}^{26}$. Adicionalmente, o uso do dispositivo como facilitador da marcha na melhora do equilíbrio pode resultar em um melhor desempenho individual no TUG. Porém, considerando que o paciente já não anda sem auxílio, ele, ainda assim, terá dificuldade de locomoção, sua mobilidade será mais lenta e seu tempo no TUG maior. 


\section{Tarefas posturais e mobilidade funcional}

O teste de correlação de Pearson analisou a associação entre as tarefas da PASS e o TUG, sendo possível hierarquizá-las em ordem decrescente de dificuldade, dispondo-as em dois grupos. No primeiro grupo, incluem-se as tarefas mais difíceis, relativas ao domínio MUP, que apresentaram correlação negativa moderada. O segundo grupo compreende as tarefas mais fáceis, de fraca correlação.

Tarefas do controle postural estático

No primeiro grupo, estão as tarefas (1) em pé sobre a perna hemiparética e (2) em pé sobre a perna não hemiparética, ambas do domínio MAP. Nossos achados, ao mostrarem correlação moderada e maior significância estatística para estas tarefas, corroboram os outros estudos ${ }^{19,20}$, que as reportaram como tarefas de mais difícil execução. No estudo original, $67 \%$ dos pacientes pontuaram - nível mais baixo na tarefa em pé sobre a perna hemiparética, sendo referida como a tarefa mais difícil ${ }^{19}$. O estudo brasileiro reportou a mesma dificuldade, assim como observado no presente estudo 20 .

Ambas as tarefas citadas estão relacionadas com 0 desempenho do controle postural em condição unipodal e requerem um único membro inferior para sustentar o peso do corpo, mantendo-se o CG alinhado verticalmente sobre a base de suporte. São posturas importantes na marcha, que estão presentes no TUG, que vão permitir a passagem pelo 
apoio médio, com distribuição equilibrada das forças internas e externas que atuam no corpo, e possibilitar o posterior avanço do membro contralateral.

Pacientes com AVC tendem a apresentar hemiparesia e consequente assimetria nos passos, desigualdade na distribuição do peso, redução do balanço dos membros superiores, sobretudo, no membro parético, além de desalinhamentos de tronco, feitos na tentativa de reestabelecer 0 equilíbrio postural, desenvolvendo estratégias biomecânicas para compensar os déficits de força muscular no membro inferior parético ${ }^{27}$.

Tarefas do controle postural dinâmico

O segundo grupo de tarefas compreende sete tarefas, dispostas em ordem decrescente de dificuldade: (3) em pé e apanhar uma caneta, (4) de sentado para a posição de pé, (5) de pé para sentado, (6) de sentado para supina, (7) em pé sem apoio, (8) de sentado para supina, e (9) de supina para o lado não afetado. São tarefas do domínio MUP, exceto a tarefa 7, que é do domínio MAP.

O domínio MUP está implicado ao deslocamento do CG sobre a base de suporte em movimento, ao controle do equilíbrio dinâmico durante a mudança de postura. Todas as tarefas do segundo grupo demandam habilidades, que estão presentes no TUG, como sentar, levantar, estar em pé em frente à cadeira, fazer meio giro e sentar.

Neste segundo grupo de tarefas, a tarefa dinâmica mais difícil observada em nosso estudo foi em pé e apanhar uma 
caneta no chão. Trata-se de uma tarefa que avalia reação de proteção $^{19}$ e que demanda ação coordenada do corpo, controle motor seletivo, flexibilidade ativa, força muscular e boa distribuição do peso sobre os membros inferiores, que devem sustentar o tronco, com adequada estabilidade para se mover e permitir o movimento do membro superior com liberdade para o alcance do objeto no chão e, posteriormente, retornar à posição original. Esta tarefa foi a que teve a mais baixa pontuação dentre as tarefas do domínio MUP, de modo que este achado mostra-se alinhado ao estudo original ${ }^{19}$.

A tarefa em pé, apanhar uma caneta no chão ainda demonstra clara participação do sistema visual como adjuvante do CP para a mobilidade. Uma revisão sistemática verificou fatores na linha de base em uma semana após AVC para identificar os potenciais preditores para a recuperação da mobilidade pós-AVC em 30 dias e incluiu a presença de hemianopsia como um dos preditores para a alta hospitalar ${ }^{28}$. Apanhar um objeto no chão implica ver o objeto e a posição que ele ocupa no espaço, o que auxilia nos constantes ajustes posturais para manutenção da postura orientada à tarefa por meio de mecanismos de feedforward e feedback, neste caso, visual.

Tarefas dinâmicas na posição sentada

Recomenda-se que pacientes com AVC agudo internados em unidades de AVC desenvolvam atividades precoces fora do leito com o objetivo de melhorar o tempo 
de deambulação sem auxílio e as $\mathrm{AVDs}^{29}$. A mobilização precoce iniciada a partir das primeiras 24 horas previne complicações secundárias do AVC, da hospitalização e da imobilização, favorece a recuperação mais rápida, a alta hospitalar, a maior independência nas AVDs e a redução do $R Q$, demonstrando melhor prognóstico e melhora da capacidade funcional e da qualidade de vida ${ }^{29}$.

No presente estudo, as tarefas até então analisadas dizem respeito ao suporte do peso do corpo sobre os membros inferiores e à permanência fora do leito, e, na sua maioria, mostram importância expressiva do $\mathrm{CP}$ estático em pé para MF. Entretanto, as tarefas posturais que permitem a saída e o retorno ao leito são dinâmicas e envolvem a participação da postura sentada. Tomando-se como exemplo referências comuns inseridas no ambiente padrão, são posturas importantes para deitar e levantar de uma cama, bem como sentar e levantar de uma da cadeira.

Uma observação atenta dos nossos resultados permitiu identificar que, após as posturas do domínio MUP, anteriormente descritas, a tarefa de sentado para de pé apesentou maior intensidade de associação entre as variáveis, seguida da postura de pé para sentado. Tarefas desse tipo, como sentar e levantar, são realizadas por meio do deslocamento do CG sobre uma base de suporte em movimento, a qual é transferida de um ponto a outro, até que se atinja o equilíbrio estático na postura final, sendo que qualquer desequilíbrio durante a transição pode desencadear queda. Em tese, quanto mais rápida e ampla a excursão do 
CG no tempo e no espaço, e menor a base de suporte final, maior a possiblidade de instabilidade postural e de RQ. Considerando-se que pacientes pós-AVC tendem a apresentar déficits no $\mathrm{CP}$, tanto a instabilidade postural, como o RQ, pode aumentar substancialmente durante as transições posturais.

Em nosso estudo, as tarefas de sentado para de pé e de pé para sentado são exemplos de tarefas que demandam força de grandes grupos musculares, ressaltando-se a maior dificuldade observada no movimento realizado contra a gravidade. Entretanto, o mesmo não foi verificado em relação às tarefas de sentado para supina e de decúbito para sentado, uma vez que o princípio da gravidade não se aplicou de igual maneira. Nossos achados demonstram que outras alterações, além da força muscular, podem contribuir para a redução da capacidade de transferência postural independente em pacientes com AVC agudo no ambiente hospitalar, implicando maior dificuldade em posturas dinâmicas que envolvem o sentar e o levantar.

Uma ampla revisão sobre quedas em pacientes com AVC mostrou que perturbações de equilíbrio autoinduzidas podem estar associadas a respostas não coordenadas e cineticamente atrasadas durante movimentos de translação e de rotação, ocorrendo com frequência em pacientes com AVC em ambiente hospitalar. O controle prejudicado dos mecanismos de ajustes antecipatórios e reativos podem tornar esses pacientes mais propensos a quedas nestas transições posturais, que indivíduos com AVC crônico, 
viventes em comunidade ${ }^{7}$. Além disso, o sentar e o levantar realizados por pacientes hemiparéticos tendem a apresentar maior tempo de execução, assimetria na distribuição do peso nos membros inferiores, bem como alteração na sequência de ativação muscular.

Falhas no planejamento ou na execução dos movimentos, ou na recuperação da instabilidade durante a mudança postural, podem ter refletido em nossos achados, uma vez que foi observado valores superiores de intensidade associativa para as tarefas sentar e levantar, quando comparadas às tarefas que se seguem.

Dando continuidade à ordem sequencial de intensidade associativa e de significância estatística decrescente, as posturas de decúbito para sentado e de sentado para supina encerram as tarefas dinâmicas que passam pela posição sentada. Entretanto, entre tais posturas, está incluída, por intensidade de correlação e significância, a tarefa intermediária em pé sem apoio, relativa ao domínio MAP. Embora a autotransferência da postura de decúbito para sentado sugira maior dificuldade do que de sentado para supina, por envolver movimentos contra a gravidade, nosso estudo mostrou que esta tarefa não foi realizada por dois pacientes, apresentando um escore mínimo de zero e, em contrapartida, a tarefa de sentado para supina não foi realizada por três pacientes, mostrando o escore mínimo de dois.

Nosso estudo corrobora estudos anteriores ${ }^{19,20}$, mostrando estas tarefas com maior facilidade de execução 
em relação às demais até então descritas. Porém, olhar para tais tarefas sob a única perspectiva da força muscular pode induzir a equívocos no raciocínio clínico. Além disso, alguns fatores podem oferecer obstáculos na execução da tarefa, como, por exemplo, o uso de acesso venoso periférico para infusão de medicamentos em membro sadio, o que impede ou dificulta que a tarefa seja executada com o auxílio dos braços ou das mãos, ou mesmo por dor, resultando em uma maior pontuação. Nestas tarefas, ainda se deve considerar que o próprio paciente pode escolher seu lado preferencial ou o qual deseja se levantar e deitar no leito, uma vez que a escala é aberta a ambas possibilidades. De todo modo, todos os fatores devem ser ponderados e os riscos avaliados antes e durante a avaliação.

Em pé sem apoio é a única postura do domínio MAP que integra esse segundo grupo de tarefas que apresentou fraca correlação com a MF. É uma tarefa de especial importância para a mobilidade em pacientes com AVC, mas não mais significativa que as anteriores, como observado no presente estudo. Há evidência na literatura que demonstra moderada correlação entre esta postura e a $\mathrm{MF}^{23}$. Por outro lado, os estudos de validação da $\operatorname{PASS}^{19}$ e de validação da versão brasileira da PASS $^{20}$ reportaram pontuação máxima nesta tarefa para todos os pacientes, destacando-a como uma das tarefas de menor dificuldade.

Embora permanecer em pé sem apoio seja um importante precursor da marcha, a fraca correlação verificada nesta tarefa em nosso estudo corrobora sua 
frequente associação a uma postura de transição. Estar em pé, em posição ereta, sem apoio, evolui comumente de forma progressiva da postura sentada, seguindo-se, posteriormente, para a caminhada, pressupondo, preliminarmente, controle de tronco adequado para um bom desempenho do equilíbrio postural dinâmico.

Outro aspecto importante a ser observado diz respeito à escala PASS, cuja ordem na execução das tarefas até então analisadas não correspondeu ao nível crescente de dificuldade, como reportada nos estudos original e da validação brasileira da PASS ${ }^{-20}$. Logo, nossos achados não corroboram este fundamento como uma das três ideias principais da PASS.

Tarefas dinâmicas na posição deitada

A última postura de fraca correlação verificada em nosso estudo foi de supino para o lado não afetado. Nossos achados mostraram que dois pacientes foram auxiliados com pouca ajuda e todos os outros a fizeram de forma independente. Trata-se de uma postura do CP dinâmico, assim como a postura de supino para o lado afetado, a qual não foi observada significância estatística, nem correlação de intensidade significante.

O status do rolamento independente de pacientes com AVCI agudo admitidos na enfermaria pode prever com precisão a capacidade de deambulação na alta hospitalar ${ }^{30}$. Os pesquisadores avaliaram 341 pacientes, divididos em grupos de deambuladores (72\%) e não deambuladores 
(28\%), admitidos imediatamente após alta da UAVC e terem passado por intervenções no programa de reabilitação. Rolamento é a habilidade motora de mover-se na cama de prona para supino e vice-versa de maneira coordenada ${ }^{30}$. Trata-se de um movimento inserido na PASS dinâmica (MUP), nas tarefas de supino para o lado afetado e não afetado.

A dificuldade de realizar tal tarefa é frequentemente associada ao déficit de força muscular do lado afetado, não favorecendo o rolamento do corpo para o lado sadio, uma vez que a transferência é feita com auxílio dos membros contralaterais para atingir a postura de decúbito desejada. Por outro lado, a tarefa de supino para o lado não afetado, cujo movimento é mais favorável para o paciente hemiparético, não foi realizada de forma independente por apenas um paciente em nosso estudo.

Considerando que os escores da PASS pouco diferem entre a alta do paciente da UAVC e sua imediata admissão na enfermaria, é possível ponderar a não consistência de nossos achados aos encontrados por Huang et al. ${ }^{30}$, uma vez que dois pacientes em nosso estudo não realizaram a tarefa mais difícil presente no rolamento e, em contrapartida, deambularam com independência durante a realização do TUG.

Tarefa sentado sem apoio

A tarefa sentado sem apoio foi realizada por todos os pacientes de forma independente, de modo que a análise não 
mostrou intensidade de correlação significante, nem significância estatística, sendo um achado consistente aos encontrados na literatura. Esta tarefa é uma das primeiras aquisições motoras após o AVC, podendo apresentar um efeito teto importante quando avaliada na primeira semana após o ictus ${ }^{19}$, o que é consoante com nossos resultados. Como explicam os pesquisadores, achados como esse podem indicar limitação no uso de uma única escala pela dificuldade de avaliar equilíbrio em pacientes pós-AVC, principalmente nas primeiras semanas após o AVC ${ }^{19}$.

\section{Controle postural: pesos e medidas}

Quedas são uma complicação crescente na hospitalização de pacientes com AVC agudo, mostrando altas taxas de queda intra-hospitalar, sobretudo, associadas ao tempo de permanência e ao programa de reabilitação, apresentando maior probabilidade de ocorrência da primeira à terceira semana de reabilitação ${ }^{8}$. Pacientes acamados são submetidos a alto grau de vigilância e estão menos expostos a situações de risco. Há uma grande variabilidade nos fatores intrínsecos e extrínsecos que contribuem para a etiologia das quedas no ambiente hospitalar. Um ponto importante a considerar é o desempenho do controle postural menos automatizado nesta população ${ }^{8}$.

Nosso estudo mostra que, em nível de dificuldade, as tarefas mais difíceis estão relacionadas àquelas que envolvem o CP estático em pé, seguidas das tarefas do CP dinâmico que passam por posturas sentadas e deitadas. 
Neste contexto, nossos achados são concordantes aos estudos de Benaim et al., que pontuam as tarefas deitar e sentar como sendo as mais fáceis e as mais difíceis, aquelas que avaliam o desempenho da postura na posição ereta ${ }^{19}$.

Nosso estudo mostrou que um paciente obteve o escore mínimo PASS11, MAP3 e MUP8 na alta da UAVC, avaliado em período de até sete dias pós-ictus, resultado este que permite afirmar que este escore mínimo pode estar associado à mobilidade independente. $O$ valor preditivo da PASS para deambulação independente na alta hospitalar de pacientes com AVC agudo foi outro objetivo investigado ${ }^{30}$, mostrando que um escore na PASS estática (MAP) superior a 3,5 pontos e na PASS dinâmica (MUP) superior a 8,5 pontos, obtidos na avaliação admissional, foram preditores precisos para deambulação independente na alta hospitalar. Este estudo aponta ainda o ponto de corte para o escore total da PASS de 12,5, porém sem significância estatística.

Considerando que os escores mínimos obtidos no presente estudo foram inferiores aos encontrados por Huang et al. para as mesmas variáveis ${ }^{30}$, e que todos os pacientes apresentaram mobilidade independente, é possível afirmar que nossos achados não corroboram os valores de corte apresentados por Huang et al. Podemos ainda inferir que nossos achados talvez possam estar relacionados à rápida melhora funcional proporcionada pela trombólise, ainda que todos os pacientes, no âmbito de cada pesquisa, tenham recebido tratamentos clínicos e fisioterapêuticos de acordo com os protocolos de seus respectivos hospitais. 


\section{Controle postural, déficit neurológico e capacidade funcional}

De acordo com os resultados obtidos para a associação independente do $\mathrm{CP}$ à gravidade do $\mathrm{AVC}$ e à capacidade funcional na alta da UAVC, nossa análise mostrou fraca correlação negativa entre PASS e NIHSS e forte correlação entre PASS e Rankin. Maior gravidade do déficit neurológico foi reportada como um fator contributivo para a baixa pontuação na PASS em estágio inicial ${ }^{30}$. O NIHSS máximo em nossa amostra foi 7, obtido por apenas dois pacientes, que tiveram escores na PASS 19 e 36, sendo que a mais baixa pontuação na PASS de 11 foi associada ao NIHSS 6 .

De todo modo, todos os pacientes mostraram déficit neurológico leve ou estavam sem déficit no momento da avaliação, apresentando NIHSS $\leq 7$ e $\mathrm{mRS} \leq 3$. Assim sendo, o presente estudo permite afirmar que pacientes com NIHSS $\leq 7$ podem apresentar mobilidade independente $e$ capacidade funcional leve, moderada ou nenhuma incapacidade significante. Adicionalmente, indica que os escores NIHSS $\leq 7$ e mRS $\leq 3$ podem estar associados ao $C P$, sendo os escores mínimos iguais ou superiores a PASS11 MAP3 MUP8 prováveis indicativos de mobilidade independente (preservada ou comprometida) em pacientes avaliados em até sete dias após admissão em UAVC, apresentando de moderado a alto RQ. 


\section{CONCLUSÃO}

Nosso estudo permitiu observar que o CP impacta na MF em pacientes com AVCI agudo trombolisados internados em UAVC que apresentam mobilidade independente preservada ou comprometida, mostrando moderada implicação do CP estático e maior comprometimento em condição unipodal, sugerindo maior RQ nestes pacientes.

Algumas limitações foram identificadas, ressaltando-se evitar generalizações na interpretação dos achados. A PASS teve um efeito de teto importante, sugerindo que este instrumento pode não ser sensível para detectar diferenças no $\mathrm{CP}$ em pacientes com AVCI que recebem terapia trombolítica. Além disso, os resultados podem não ser adequados para avaliar pacientes com outros tipos de AVC ou em outras fases da doença, dada à especificidade no perfil da população estudada. A amostra reduzida pode ter conduzido à detecção de poucas variações nos resultados, sugerindo-se estudos mais amplos, com amostras mais expressivas, que possam confrontar ou corroborar os achados desta pesquisa.

Pacientes com AVCI agudo trombolisados podem apresentar melhora significativa da condição neurológica e um prognóstico favorável para o CP e a MF na alta hospitalar. Outros instrumentos complementares podem ser considerados para uma avaliação mais precisa. Adicionalmente, abordagens qualitativas dos componentes do CP e da MF podem aprofundar as interpretações dos resultados mensurados em avaliações com instrumentos 
objetivos, tendo em vista que a maior eficiência advém da capacidade de dimensionar a possível melhora das condições neurofuncionais do paciente associadas às suas prioridades.

\section{REFERÊNCIAS}

1. Horak FB. Postural orientation and equilibrium: what do we need to know about neural control of balance to prevent falls? Age Ageing 2006;2:ii7-11. http://dx.doi.org/10.1093/ageing/afl077

2.Cupps B. Postural control: a current review. Neuro Develop Treat 1997; 1:3-8.

3.Pinto EB, Nascimento C, Monteiro M, Castro M, Maso I, Campos A, et al. Proposal for a New Predictive Scale for Recurrent Risk of Fall in a Cohort of Community-Dwelling Patients with Stroke. J Stroke Cerebrovasc Dis 2016;25:2619-26.

http://dx.doi.org/10.1016/j.jstrokecerebrovasdis.2016.06.045

4.Tinetti ME, Ginter SF. Identifying Mobility Dysfunctions in Elderly Patients Standard Neuromuscular Examination or Direct Assessment? JAMA $1988 ; 259: 1190-3$.

http://dx.doi.org/10.1001/jama.1988.03720080024022

5.Chang M, Cohen-Mansfield J, Ferruci L, Leveille S, Volpato S, Rekeneire $\mathrm{N}$, et al. Incidence of loss of ability to walk 400 meters in a functionally limited older population. J Am Geriatr Soc 2004;52:20948. http://dx.doi.org/10.1111/j.1532-5415.2004.52570.x

6.Podsiadlo D, Richardson S. The Timed "Up \& Go": A test of basic functional mobility for frail elderlv persons. J Am Geriatr Soc $1991 ; 39: 142-8 . \quad \quad$ http://dx.doi.org/10.1111/j.15325415.1991.tb01616.x

7. Weerdesteyn V, Niet M, Duijnhoven Hanneke JRv, Geurts ACH. Falls in individuals with stroke. J Rehabil Res Dev 2008;45:1195-213. http://dx.doi.org/10.1682/JRRD.2007.09.0145

8. Holloway RG, Tuttle D, Baird T, Skelton WK. The safety of hospital stroke care. Neurology 2007;68:550-5.

http://dx.doi.org/10.1212/01.wnl.0000254992.39919.2e

9. Maniva SJC, Freitas CHA. Uso de alteplase no tratamento do acidente vascular encefálico isquêmico agudo: o que sabem os enfermeiros. Rev Bras Enferm 2012;65:474-81. http://dx.doi.org/10.1590/S003471672012000300012

10.Garritano CR, Luz PM, Pires MLE, Barbosa MTS, Batista KM. Analysis of the mortality trend due to cerebrovascular accident in Brazil in the XXI Century. Arq Bras Cardiol 2012;98:519-27.

http://dx.doi.org/10.1590/S0066-782X2012005000041

11.Bezerra AMS. Trombólise em pacientes com acidente vascular cerebral isquêmico agudo: aspectos epidemiológicos e funcionais na 
admissão e alta hospitalar (dissertação). Fortaleza: Universidade de Fortaleza, 2012.

https://bdtd.ibict.br/vufind/Record/UFOR 0a1c47313ff71380bd1159a 8de191c8a

12.Oliveira-Filho J. Trombólise no acidente vascular cerebral isquêmico: Uma mudança conceitual. Rev Neurocienc 2005;13:10004. http://dx.doi.org/10.34024/rnc.2005.v13.8835

13. Oliveira GMM, Brant LCC, Polanczyk CA, Biolo A, Nascimento BR, Malta DC, et al. Estatística cardiovascular - Brasil 2020. Arq Bras Cardiol 2020;115:308-439.

http://dx.doi.org/10.36660/abc.20200812

14. Brott T, Adams HP Jr, Olinger CP, Marler JR, Barsan WG, Biller J, et al. Measurements of acute cerebral infarction: a clinical examination scale. Stroke 1989;20:864-70.

http://dx.doi.org/10.1161/01.STR.20.7.864

15.Cincura C, Pontes-Neto OM, Neville IS, Mendes HF, Menezes DF, Mariano DC, et al. Validation of the National Institutes of Health Stroke Scale, modified Rankin Scale and Barthel Index in Brazil: the role of cultural adaptation and structured interviewing. Cerebrovasc Dis 2009;27:119-22. http://dx.doi.org/10.1159/000177918

16.AVERT Trial Collaboration group. Efficacy and safety of very early mobilisation within $24 \mathrm{~h}$ of stroke onset (AVERT): a randomised controlled trial. Lancet 2015;386:46-55.

http://dx.doi.org/10.1016/S0140-6736 (15) 60690-0

17.Rankinune J. Cerebral vascular accidents in patients over the age of 60. II. Prognosis. Scott Med J 1957;2:200-15.

http://dx.doi.org/10.1177/003693305700200504

18. Wilson JL, Hareendran A, Hendry A, Potter J, Bone I, Muir KW. Reliability of the Modified Rankin Scale across multiple raters: benefits of a structured interview. Stroke 2005;36:777-81.

http://dx.doi.org/10.1161/01.STR.0000157596.13234.95

19.Benaim C, Pérennou DA, Villy J, Rousseaux M, Pelissier JY. Validation of a standardized assessment of postural control in stroke patients: the Postural Assessment Scale for Stroke Patients (PASS). Stroke 1999;30:1862-8. http://dx.doi.org/10.1161/01.str.30.9.1862 20.Yoneyama SM, Roiz RM, Oliveira TM, Oberg TD, Lima NMFV. Validação da versão brasileira da Escala de Avaliação Postural para Pacientes após Acidente Vascular Encefálico. Acta Fisiatr 2007;15:96100.

https://www.revistas.usp.br/actafisiatrica/article/view/102920/10122 $\underline{3}$

21.Andersson AG, Kamwendo K, Seiger A, Appelros P. How to identify potential fallers in a stroke unit: validity indexes of 4 test methods. J Rehabil Med 2006;38:186-91.

http://dx.doi.org/10.1080/16501970500478023

22. Mukaka MM. Statistics Corner: A guide to appropriate use of Correlation coefficient in medical research. Malawi Med J 2021;24:6971. https://www.ncbi.nlm.nih.gov/pmc/articles/PMC3576830/ 
23. Antunes J, Justo FHO, Justo AFO, Ramos GC, Prudente CM. Influência do controle postural e equilíbrio na marcha de pacientes com sequela de acidente vascular cerebral. Rev Fisioter S Fun 2016;5:3041.

http://www.periodicos.ufc.br/fisioterapiaesaudefuncional/article/down load/20607/31043

24.Mao HF, Hsueh IP, Tang PF, Sheu CF, Hsieh CL. Analysis and comparison of the psychometric properties of three balance measures for stroke patients. Stroke 2002;33:1022-7. http://dx.doi.org/10.1161/01.str.0000012516.63191.c5

25. Johansen LK, Stistrup RD, Schjøtt CS, Madsen J, Vinther A. Absolute and Relative Reliability of the Timed 'Up \& Go' Test and '30second Chair-Stand' Test in Hospitalised Patients with Stroke. PLoS One 2016;11:e0165663. http://dx.doi.org/10.1371/journal.pone.0165663 26.Peter KJ, Furie KL, Shafqat S, Rallis N, Chang Y, Stein J. Functional recovery following rehabilitation after hemorrhagic and ischemic stroke. Arch Phys Med Rehabil 2003;84:968-72.

http://dx.doi.org/10.1016/s0003-9993 (03) 00040-6

27. Chaves CM. Aplicação do TUG-ABS Português-Brasil para avaliação funcional de indivíduos com hemiparesia pós-AVE com diferentes níveis de mobilidade. Conexão Ci 2017;12:37-46.

https://doi.org/10.24862/cco.v12i2.596

28.Craig LE, Wu O, Bernhardt J, Langhorne P. Predictors of poststroke mobility: systematic review. Int J Stroke 2011;6:321-7. http://dx.doi.org/10.1111/j.1747-4949.2011.00621.x

29.Cumming TB, Thrift AG, Collier JM, Churilov L, Dewey HM, Donnan $\mathrm{GA}$, et al. Very early mobilization after stroke fast-tracks return to walking: further results from the phase II AVERT randomized controlled trial. Stroke 2011;42:153-8.

http://dx.doi.org/10.1161/STROKEAHA.110.594598

30. Huang YC, Wang WT, Liu TH, Liao CD, Lin LF, Huang S. Postural Assessment Scale for Stroke Patients Scores as a predictor of stroke patient ambulation at discharge from the rehabilitation Ward. J Rehabil Med 2016;48:259-64. http://dx.doi.org/10.2340/16501977-2046 